\title{
Identification of Amplified Fragment Length Polymorphism Markers Linked to Gummy Stem Blight (Didymella bryoniae) Resistance in Melon (Cucumis melo L.) PI 420145
}

\author{
Joseph N. Wolukau, Xiaohui Zhou, and JinFeng Chen ${ }^{1}$ \\ State Key Laboratory of Crop Genetics and Germplasm Enhancement, \\ Nanjing Agricultural University, Tongwei Lu 6\#, Nanjing, Jiangsu 210095, \\ China
}

Additional index words. gummy stem blight, bulk segregant analysis, marker-assisted selection

\begin{abstract}
Gummy stem blight (GSB) caused by the ascomycete fungus Didymella bryoniae (Auersw.) Rehm is an important disease of melon. Molecular markers linked to resistance would be useful for melon breeding programs. The amplified fragment length polymorphism (AFLP) technique and bulk segregant analysis were used to identify molecular markers linked to the resistance of melon to Didymella bryoniae. Segregation analysis of $F_{2}$ progeny from a cross of PI 420145, a resistant line, and PI 136170, a susceptible line, showed that resistance to GSB was controlled by a dominant gene. One AFLP marker, E-TG/M-CTC ${ }_{200}$, was identified that is tightly linked to GSB resistance gene at a distance of $2.0 \mathrm{cM}$. To our best knowledge, this is the first report of AFLP markers linked to GSB resistance in melon. The identification of AFLP markers provides a step toward the use of marker-assisted selection and the characterization of the gene encoding resistance to GSB in melon.
\end{abstract}

Gummy stem blight (GSB) caused by Didymella bryoniae (Auersw.) Rehm is one of the most important soilborne diseases of melon and causes significant economic losses (30\% to $60 \%$ ) (Frantz and Jahn, 2004). Although chemical control has been successfully used to manage GSB, repeated use of fungicides is not advisable because of the negative impact on the environment. In fact, development of resistance to some systemic benzimidazole fungicides used against $D$. bryoniae has been reported from several cucurbit production areas (Keinath and Zitter, 1998). Crop rotations are only partially effective, because airborne inoculum coming in from other areas can help to initiate new infections (Tullu et al., 2002). Therefore, use of resistant cultivars can be considered as the most environmentally sustainable and economical means of GSB disease management.

Received for publication 31 July 2008. Accepted for publication 23 Oct. 2008.

This work was partially supported by Program no. 2005-2007 from Jiangsu Natural Science Foundation, the '863' Programs (2006AA10Z1A8, 2006AA100108).

The suggestions of four anonymous reviewers to revise the manuscript and the modifying by Ahmed Abbas Malik are greatly appreciated.

The authors Joseph N. Wolukau and Xiaohui Zhou contributed equally to this paper.

${ }^{1}$ To whom reprint requests should be addressed; e-mail jfchen@njau.edu.cn.
Several sources of resistance to GSB have been reported; however, most GSB-resistant melon varieties and breeding lines released to date have been derived from a resistant accession, PI 140471 (McGrath et al., 1993; Norton et al., 1985; Sowell et al., 1966), and have failed to provide adequate levels of resistance (Zhang et al., 1997). Inheritance studies showed that one dominant gene $(M c)$ confers resistance to GSB in PI 140471 (Sowell et al., 1966). Frantz and Jahn (2004) reported that PIs 157082, 511890, and 482398 carry a monogenic dominant gene for GSB resistance, whereas resistance in PI 482399 was monogenic-recessive. The five independent genes in PIs 140471, 157082, 511890, 482498, and 482399 have been renamed as $G s b-1, G s b-2, G s b-3, G s b-$ 4 , and $g s b-5$, respectively (Frantz and Jahn, 2004). Recently, we reported that the resistance to GSB in PI 420145 is conferred by a single dominant gene and the resistant level of PI 420145 was found to be equal to or even greater than the five accessions (Wolukau et al., 2007). However, allelism in this accession has not been conducted and the gene has not been named.

Selection for resistance to GSB is of pivotal importance to melon breeding programs. Phenotypic selection is tedious and sensitive to environmental conditions. Identification of linked markers is an essential step toward both marker-assisted selection (MAS) and map-based cloning. MAS increases selection efficiency not only by allowing for earlier selection, but also by reducing plant population size during breeding without phenotypic of progeny (Wang and Roberts, 2006). Markers linked to different genes for resistance can also be used to pyramid resistance genes into commercially acceptable cultivars.

Amplified fragment length polymorphism (AFLP) markers combined with bulk segregant analysis (BSA) is a powerful technique for identifying markers linked to resistance genes. Kwon et al. (1998) explored linkages between the GSB resistance and AFLP markers in watermelon. However, there has been no report of molecular markers linked to GSB resistance in melon. In the present study, experiments were designed to identify AFLP markers linked to GSB resistance in PI 420145.

\section{Materials and Methods}

Plant and fungal material. PI 420145 (Cucumis melo subsp. melo) was originally collected from Japan and has resistance to $D$. bryoniae under field, greenhouse, and plastic tunnel conditions. It has moderate-sized green fruits, larger than those of PI 140471, and could easily be deployed into commercial germplasms (Wolukau et al., 2007; Zhang et al., 1997). PI 136170 (Cucumis melo subsp. melo), originally collected from Italy, was used as the susceptible parent. Both PIs were kindly supplied by USDA, NPGS Ames, IA, and appeared well adapted to our conditions. Spore isolates (IS25) of Didymella bryoniae were obtained from diseased melon plant materials in plastic tunnels in Nanjing, China. Inoculum was prepared according to Kwon et al. (1997) as modified by Wolukau et al. (2007).

Disease tests for gummy stem blight resistance. The resistance tests were performed according to Zhang et al. (1997). Uniformly germinated seedlings were transplanted into $12 \mathrm{~cm} \times 13-\mathrm{cm}$ plastic pots filled with sand, soil, and vermiculite in a ratio of $1: 1: 1$. Resistant and susceptible accessions were placed randomly among the pots as disease severity checks and to aid in disease spread. Plants were inoculated at the four to six true leaf stage. After inoculation, plants were covered with a tight plastic tent within the tunnel to maintain high relative humidity. The plastic cover was removed $3 \mathrm{~d}$ after inoculation. Visual ratings for the appearance of disease on stems were scored at 7, 14, and $21 \mathrm{~d}$ postinoculation using the following scale: 1 , no damage; 2 , single lesion 1 to $10 \mathrm{~mm}$ long or composite lesions 1 to $20 \mathrm{~mm}$, stem not girdled; 3 , lesion 21 to $80 \mathrm{~mm}$ long and/or girdling of stem; 4 , stem withered; and 5 , seedling dead. Plants that received a rating of 1 or 2 were considered resistant and those greater than 3 were susceptible.

DNA isolation, bulk segregant analysis, and amplified fragment length polymorphism analysis. DNA was extracted by a modified cetyltrimethyl ammonium bromide method based on Murray and Thompson (1980). For BSA (Michelmore et al., 1991), equivalent 
amounts of genomic DNA from 10 resistant and 10 susceptible $F_{2}$ plants were pooled to form resistant and susceptible bulks. Both bulks were used along with the parents to identify markers showing polymorphisms between the four samples. AFLP analysis was performed as described by Vos et al. (1995) with the following modifications: $300 \mathrm{ng}$ DNA of each sample was digested with EcoRI/MseI restriction enzymes and the digested fragments were ligated with EcoRI and MseI adaptors, respectively. The preamplification product was diluted in a ratio of 1:20 with $\mathrm{ddH}_{2} \mathrm{O}$ and used as a template for selective amplification with $E c o R I+2$ (two selective bases) and $M s e I+3$ (three selective bases) primers. The selective amplification thermal cycling profile was as follows: 11 cycles at $94{ }^{\circ} \mathrm{C}$ for $30 \mathrm{~s}, 65^{\circ} \mathrm{C}$ for $30 \mathrm{~s}$, and $72{ }^{\circ} \mathrm{C}$ for $1 \mathrm{~min}$, followed by 23 cycles at $94{ }^{\circ} \mathrm{C} 30 \mathrm{~s}, 56{ }^{\circ} \mathrm{C}$ for $30 \mathrm{~s}$, and $72^{\circ} \mathrm{C}$ for $1 \mathrm{~min}$ and then recovered at $4{ }^{\circ} \mathrm{C}$. The gels were stained with $\mathrm{AgNO}_{3}$ solution according to the method described by Panaud et al. (1996). Each amplification and electrophoresis was repeated once. Repeatable banding patterns were scored as presence (1) or absence ( 0$)$ of a DNA band. DNA fragment sizes were estimated by comparison of migration distance to that of a 100-bp DNA ladder. Fragments were designated following the AFLP marker nomenclature described by Ouedraogo et al. (2001). Chi square analyses were performed to ascertain the goodness of fit between the expected ratio for a single dominant gene and the segregation of the phenotypic data. Linkage analysis between the AFLP markers and the GSB resistance loci was performed with Map Maker (Version 3.0; Whitehead Institute, Cambridge, MA) software. Correlations between the resistance phenotype and the GSB-linked markers were analyzed using PROC CORR procedure of the SAS (Version 6.0; SAS Institute, Cary, NC).

\section{Results and Discussion}

The inheritance test for resistance of $F_{2}$ individuals to GSB before the molecular investigations was conducted. Among the 89 individuals of $F_{2}$ population derived from the cross between PI 420145 and PI 136170, 69 were scored as resistant and 20 were susceptible. The segregation fits a $3: 1$ ratio (resistant to susceptible, $\chi^{2}=0.30, P=0.55$ ) to GSB, indicating that resistance to GSB in PI 420145 is monogenic-dominant.

To identify markers associated with GSB resistance, a total of 64 AFLP primer combinations (Table 1) were used to screen DNA from the two parental lines and two bulks constructed for the BSA analysis. Thirty-six (56.25\%) primer combinations showed polymorphism between the parents and the bulks. Twelve (18.75\%) (E-AA/M-CTC, E-AC/MCC, E-AT/M-CAT, E-AT/M-CTG, E-TA/MCAC, E-TC//M-CAG, E-TG/M-CAC, E-TG/ M-CAG, E-TG/M-CAT, E-TG/M-CTA, E-TG/M-CTC, and E-TG/M-CTG) were specific for GSB resistance. These 12 combinations were tested on the bulked individuals to

Table 1. Primers used in the screening for amplified fragment length polymorphism markers linked to the resistance to gummy stem blight (Didymella bryoniae) using $\mathrm{F}_{2}$ progenies of the cross of PI $420145 \times$ PI 136170 .

\begin{tabular}{|c|c|c|c|}
\hline \multicolumn{4}{|c|}{ Preamplification } \\
\hline $\mathrm{EOO}$ & 5'-GACTGCGTACCAATTC-3' & MOO & 5'-GATGAGTCCTGAGTAA-3' \\
\hline \multicolumn{4}{|c|}{ Selective amplification } \\
\hline E-AA & 5'-GACTGCGTACCAATTCAA-3' & M-CAA & 5'-GATGAGTCCTGAGTAACAA-3' \\
\hline E-AC & 5'-GACTGCGTACCAATTCAC-3' & $\mathrm{M}-\mathrm{CAC}$ & 5'-GATGAGTCCTGAGTAACAC-3' \\
\hline E-AG & 5'-GACTGCGTACCAATTCAG-3' & $\mathrm{M}-\mathrm{CAG}$ & 5'-GATGAGTCCTGAGTAACAG-3' \\
\hline E-AT & $5^{\prime}$-GACTGCGTACCAATTCAT-3' & M-CAT & 5'-GATGAGTCCTGAGTAACAT-3' \\
\hline E-TA & 5'-GACTGCGTACCAATTCTA-3' & M-CTA & 5'-GATGAGTCCTGAGTAACTA-3' \\
\hline E-TC & 5'-GACTGCGTACCAATTCTC-3' & M-CTC & $5^{\prime}$-GATGAGTCCTGAGTAACTC-3' \\
\hline E-TG & 5'-GACTGCGTACCAATTCTG-3' & M-CTG & 5'-GATGAGTCCTGAGTAACTG-3' \\
\hline E-TT & 5'-GACTGCGTACCAATTCTT-3' & M-CTT & 5'-GATGAGTCCTGAGTAACTT-3' \\
\hline
\end{tabular}

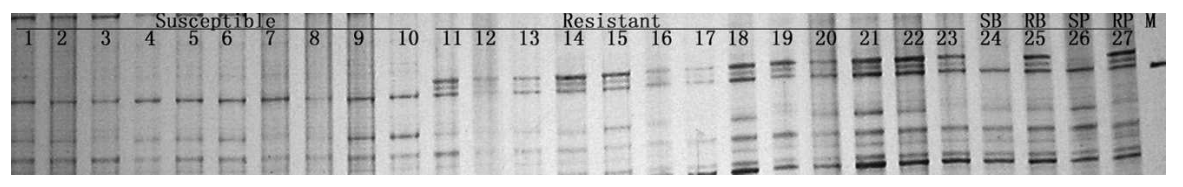

Fig. 1. Amplification of genomic DNA from $\mathrm{F}_{2}$ progenies of PI $420145 \times$ PI 136170 using the E-TG/M$\mathrm{CTC}_{200}$ amplified fragment length polymorphism marker. Lanes 1 through 10 are individuals in the susceptible bulk; lanes 11 through 23 are individuals in the resistant bulk; lane 24 is the susceptible bulk; lane 25 is the resistant bulk; lane 26 is the susceptible parent; and lane 27 is the resistant parent, where lane $\mathrm{M}$ is the 100-bp ladder.

eliminate false-positive markers and four primer pairs (E-TG/M-CTC, E-AT/M-CTG, E-TC/M-CAG, and E-TG/MCTA) were identified to have potential linkage to the GSB resistance. These markers were then used to screen $\mathrm{F}_{2}$ individuals and one marker (E-TG/M-CTC) was found to be tightly linked to the GSB resistance (Fig. 1). Primer combination E-TG/M-CTC showed an $\approx 200$-bp amplification product present in the resistant parent PI 420145, the resistant bulk, and 68 of 69 resistant individuals but absent in the susceptible parent, the susceptible bulk, and 20 susceptible individuals. The correlation between the marker and GSB resistance is 0.905 at a $P$ value of 0.01 , indicating that this marker is associated with resistance to GSB. The marker was found to be linked to the GSB resistance gene at a distance of $2.0 \mathrm{cM}$ and designated as E-TG/ $\mathrm{M}-\mathrm{CTC}_{200}$.

Markers tightly linked with GSB resistance gene could be useful in MAS. However, markers associated with $D$. bryoniae resistance have not been previously reported in melon (C. melo L.). Kwon et al. (1998) explored linkage between the GSB resistance locus and AFLP markers in watermelon, but their result was not conclusive. In the present study, AFLP markers linked to the D. bryoniae resistance in PI 420145 were developed. This marker can be used to tag the resistance gene and facilitate marker-assisted selection for resistance to GSB in segregating populations of melon using PI 420145 as the source of resistance.

Several GSB-resistant melon genotypes have been shown to carry monogenic independent dominant genes for resistance such as $G s b-1, G s b-2, G s b-3, G s b-4$, and $g s b-5$ (Frantz and Jahn, 2004). However, it not known if the dominant gene in PI 420145 is allelic to the previously mentioned genes. Allelism tests are currently underway and that should resolve the relationship between the resistance allele(s) present in PI 420145 and other GSB-resistant PI. It is possible that the resistance gene in PI 420145 may be allelic to one or more of the genes in other GSB-resistant PIs and in such a situation, some of the GSB resistance markers described in our study might be useful to analyze segregating melon populations generated from other PIs.

\section{Literature Cited}

Frantz, J.D. and M.M. Jahn. 2004. Five independent loci each control monogenic resistance to gummy stem blight in melon (Cucumis melo L.). Theor. Appl. Genet. 108:1033-1038.

Keinath, A.P. and T.A. Zitter. 1998. Resistance to benomyl and thiophanate-methyl in Didymella bryoniae from South Carolina and New York. Plant Dis. 82:479-484.

Kwon, M.K., J.R. Hong, H.J. Sun, K.Y. Sung, B.H. Cho, and K.C. Kim. 1997. Standardization of a mass production technique for pycnidiospores of Didymella bryoniae, gummy stem blight fungus of cucurbits. Korean J. Plant Pathol. 13:105-112.

Kwon, Y.S., Y.H. Om, L. Hawkins, and F. Dane. 1998. Molecular tagging of gummy stem blight resistance in watermelon. HortScience 33: 472 .

McGrath, D.J., L. Vawdrey, and O. Walker. 1993. Resistance to gummy stem blight in muskmelon. HortScience 28:930-931.

Michelmore, R., I. Paran, and K. Kesseli. 1991. Genetic mapping of $P h-2$, a single locus controlling partial resistance to Phytophthora infestans in tomato. Mol. Plant Microbe Interact. 11:259-269.

Muray, H.G. and W.F. Thompson. 1980. Rapid isolatioin of higher weight DNA. Nucleic Acids Res. 8:4321-4325.

Norton, J.D., R.D. Cosper, D.A. Smith, and K.S. Rymal. 1985. Aurora-A high quality disease resistant cantaloupe. Alabama Agr. Expt. Sta. Circ. 278.

Ouedraogo, J.T., V. Maheshwari, D.K. Berner, C.A. St. Oierre, F. Belzile, and M.P. Timko. 
2001. Identification of AFLP markers linked to resistance of cowpea (Vignia unguiculata L.) to parasitism by Stiga gesnerioides. Theor. Appl. Genet. 102:1029-1036.

Panaud, O., X. Chen, and S.D. McCouch. 1996. Development of microsatellite markers and characterization of simple sequence length polymorphism (SSLP) in rice (Oryza sativa L.). Mol. Gen. Genet. 252:597-607.

Sowell, G., Jr., K. Prasad, and J.D. Norton. 1966. Resistance of Cucumis melo introductions to Mycosphaerella citrullina. Plant Dis. Rpt. 50:661-663.
Tullu, A., L. Bachwaldt, T. Warkentin, B. Taran, and A. Vandenberg. 2002. Genetics of resistance to anthracnose and identification of AFLP and RAPD markers linked to the resistance gene in PI 320937 germplasm of lentil (Lens culinaris Medikus). Theor. Appl. Genet. 106:428-434.

Vos, P., R. Hogers, M. Bleeker, M. Reijans, T. van de Lee, M. Hornes, A. Frijters, J. Pot, J. Peleman, M. Kuiper, and M. Zabeau. 1995. AFLP: A new technique for DNA fingerprinting. Nucleic Acids Res. 23:4407-4414.

Wang, C.L. and P.A. Roberts. 2006. Development of AFLP and derived CAPS markers for root- knot nematode resistance in cotton. Euphytica 152:185-196.

Wolukau, J.N., X.H. Zhou, Y. Li, Y.B. Zhang, and J.F. Chen. 2007. Resistance to gummy stem blight in melon (Cucumis melo L.) germplasm and inheritance of resistance from Plant Introductions 157076, 420145, and 323498. HortScience 42:215-221.

Zhang, Y.P., M.M. Kyle, K. Anagnostou, and T.A. Zitter. 1997. Screening melon (Cucumis melo L.) for resistance to gummy stem blight in the greenhouse and field. HortScience 32:117121. 